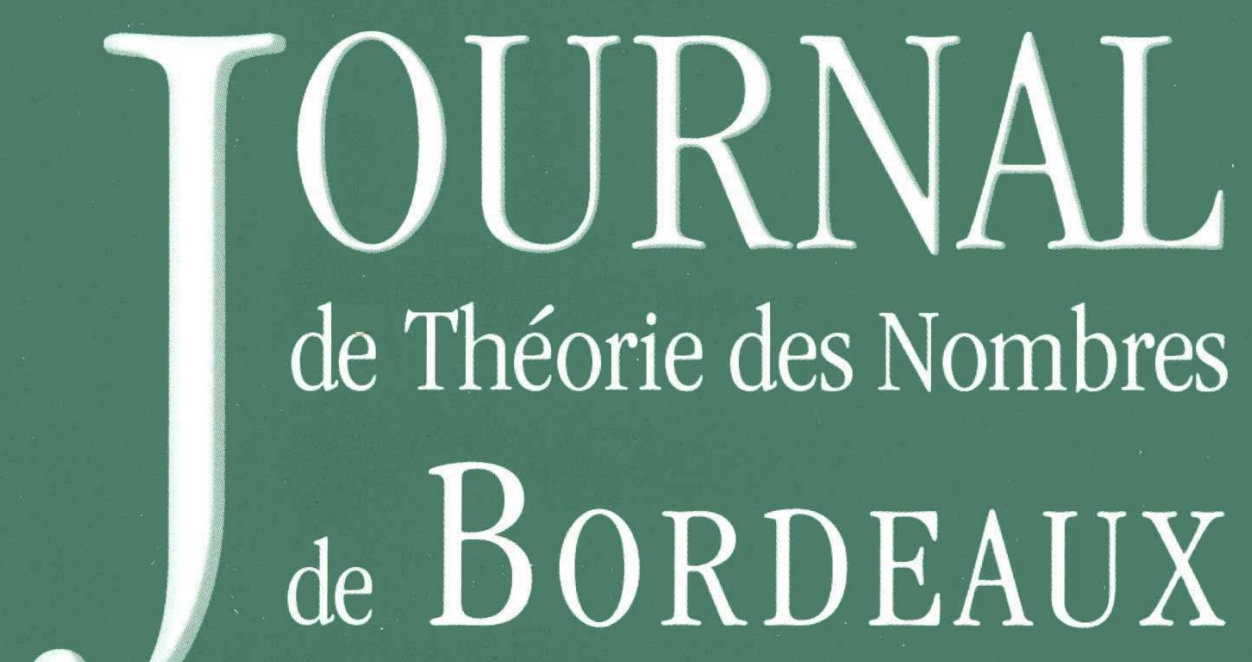

anciennement Séminaire de Théorie des Nombres de Bordeaux

Ehud DE SHALIT

Mahler bases and elementary $\boldsymbol{p}$-adic analysis

Tome 28, no 3 (2016), p. 597-620.

<http://jtnb.cedram.org/item?id=JTNB_2016__28_3_597_0>

(C) Société Arithmétique de Bordeaux, 2016, tous droits réservés.

L'accès aux articles de la revue «Journal de Théorie des Nombres de Bordeaux » (http://jtnb.cedram.org/), implique l'accord avec les conditions générales d'utilisation (http://jtnb.cedram. org/legal/). Toute reproduction en tout ou partie de cet article sous quelque forme que ce soit pour tout usage autre que l'utilisation à fin strictement personnelle du copiste est constitutive d'une infraction pénale. Toute copie ou impression de ce fichier doit contenir la présente mention de copyright.

\title{
cedram
}




\title{
Mahler bases and elementary $p$-adic analysis
}

\author{
par EHUD DE SHALIT
}

\begin{abstract}
RÉSUMÉ. Divers théorèmes d'analyse $p$-adique élémentaires, souvent prouvés uniquement sur $\mathbb{Q}_{p}$ (cf. [4]), sont généralisés à tout corps local, avec l'aide de la notion de base de Mahler. Dans la dernière section nous expliquons la relation entre nos résultats et ceux de [10].
\end{abstract}

ABSTRACT. Various theorems in elementary $p$-adic analysis, commonly proved only for $\mathbb{Q}_{p}$, and summarized in [4], are generalized to any local field, using the concept of a Mahler basis. In the last section we explain the relation of these results to the work [10]. The paper is largely self-contained.

\section{Mahler bases}

1.1. Background. Fix a prime number $p$, let $\mathbb{Z}_{p}$ denote the ring of $p$ adic integers, and $\mathbb{Q}_{p}$ its fraction field. Mahler's theorem [6] states that the binomial coefficients $\left(\begin{array}{l}x \\ n\end{array}\right)(n \geq 0)$ form an orthonormal basis for the $p$-adic Banach space $C\left(\mathbb{Z}_{p}, \mathbb{Q}_{p}\right)$ of continuous $\mathbb{Q}_{p}$-valued functions on $\mathbb{Z}_{p}$. In other words, every continuous function $f$ from $\mathbb{Z}_{p}$ to $\mathbb{Q}_{p}$ has a unique uniformly convergent expansion

$$
f=\sum_{n=0}^{\infty} a_{n}\left(\begin{array}{l}
x \\
n
\end{array}\right)
$$

where $a_{n} \rightarrow 0$, and $|f|_{\text {sup }}=\max \left|a_{n}\right|$. Conversely, every such expansion defines a continuous function.

A fact closely related to Mahler's theorem is that the functions $\left(\begin{array}{l}x \\ n\end{array}\right)$ $(n \geq 0)$ form an algebraic basis for the $\mathbb{Z}_{p}$-module $\operatorname{Int}\left(\mathbb{Z}_{p}\right)$ of polynomials over $\mathbb{Q}_{p}$ which are integral valued on $\mathbb{Z}_{p}$.

Various subspaces of $C\left(\mathbb{Z}_{p}, \mathbb{Q}_{p}\right)$ can be described by the asymptotic behavior of the Mahler coefficients $a_{n}$. For example, $f$ is Lipschitz if and only if $\inf \left(v\left(a_{n}\right)-\log _{p} n\right)>-\infty$, where $v$ is the $p$-adic valuation, and $f$ is locally analytic if and only if $v\left(a_{n}\right)-\varepsilon n \rightarrow \infty$ for some $\varepsilon>0$.

Manuscrit reçu le 25 juin 2014, révisé le 7 novembre 2014, accepté le 21 novembre 2014.

Mathematics Subject Classification. 11S31, 11S80.

Mots-clefs. Mahler's theorem, Amice transform, locally analytic distributions, Lubin-Tate groups. 
The Banach dual of $C\left(\mathbb{Z}_{p}, \mathbb{Q}_{p}\right)$ is the space of $p$-adic measures on $\mathbb{Z}_{p}$, and is well known to be isomorphic to the Iwasawa algebra $\mathbb{Q}_{p} \otimes \mathbb{Z}_{p}[[T]]$, convolution of measures corresponding to the usual multiplication of power-series. It is instructive to think of the Iwasawa algebra as the algebra of bounded rigid analytic functions on the open unit disk $\Delta$. In the same vein, the algebra of locally analytic distributions is isomorphic to the algebra of all rigid analytic functions on $\Delta$ (a result of Amice, which is equivalent to the characterization of locally analytic functions by their Mahler coefficients, mentioned above).

In this note we study the extensions of these results to the ring of integers of an arbitrary local field. Some of the results are not new, but for the sake of completeness and uniformity we have included them as well.

Let $K$ be a local field, $R$ its ring of integers, and $\pi$ a uniformizer. Let $q$ be the cardinality of the residue field $\kappa=R / \pi R$, and $p=\operatorname{char}(\kappa)$. The field $K$ may be of characteristic 0 or $p$. We normalize the discrete valuation $v$ on $K^{\times}$ so that $v(\pi)=1$. We let $\mathcal{K}$ be the completion of a fixed separable closure of $K$ (when $K$ is of characteristic 0 this is the field commonly denoted $\mathbb{C}_{p}$ ).

We denote by $C(R, K)$ the $K$-Banach space of continuous functions from $R$ to $K$, with the sup norm. We denote by $\operatorname{Int}(R)$ the $R$-module of polynomials over $K$ which are integral valued on $R$. Both $C(R, K)$ and $\operatorname{Int}(R)$ have a ring structure, but until much later (cf. Section 6 ) we shall only be concerned with their additive structure. In Sections 1-4 we study the Mahler expansion of an $f \in C(R, K)$ and characterize various subspaces of $C(R, K)$ by means of the asymptotics of Mahler coefficients. In contrast to the classical case, there is no canonical choice of a Mahler basis such as the basis of binomial coefficients, so we axiomatize the concept of a Mahler basis and work with an arbitrary one.

This lack of a canonical basis prevents the analogue of the Iwasawa-Amice transform from being an isomorphism between algebras of measures (or distributions) and rings on functions on $\Delta$. It is only an additive isomorphism. We explain this in Section 5. In a remarkable paper [10] Schneider and Teitelbaum discovered how to remedy this, at the expense of introducing a rigid analytic curve $\tilde{\Delta}$ which is a twisted version of $\Delta$. Let $L(R, K)$ be the space of locally $K$-analytic functions on $R$, topologized as in Section 4.1. The space of locally analytic distributions $L(R, K)^{\prime}$ is its topological dual. The main result of [10] is a canonical isomorphism of algebras between $L(R, K)^{\prime}$ and the ring of rigid analytic functions on $\tilde{\Delta}$. Section 6 is a self-contained exposition of the results of Schneider and Teitelbaum. Except for Proposition 6.1 , for which we found an elementary proof, we follow their paper rather closely.

Our exposition employs Lubin-Tate groups, but in a way this is a "red herring", allowing us to present elegant proofs for some of the claims, but 
not an essential ingredient in itself. (Lubin-Tate groups will reappear in an essential way in Section 6, when we discuss the construction of Schneider and Teitelbaum.)

1.2. Mahler bases. For $n \geq 0$, let

$$
\operatorname{Int}_{n}(R)=\{f \in \operatorname{Int}(R) \mid \operatorname{deg}(f) \leq n\}
$$

and let $\mathfrak{a}_{n} \subset K$ be the fractional ideal of leading coefficients of polynomials from $\operatorname{Int}_{n}(R)$. If $f_{n} \in \operatorname{Int}(R)$ is a polynomial of degree $n$ whose leading coefficient generates $\mathfrak{a}_{n}$, then $\operatorname{Int}_{n}(R)=\operatorname{Int}_{n-1}(R) \oplus R f_{n}$. A sequence $\left\{f_{n}\right\}_{n>0}$ as above will be called a Mahler basis. Note that $f_{n}$ is unique up to multiplication by a unit and addition of an $R$-linear combination of $f_{0}, \ldots, f_{n-1}$.

For every $n \geq 1$ write

$$
w_{q}(n)=\sum_{i=1}^{\infty}\left\lfloor\frac{n}{q^{i}}\right\rfloor .
$$

Clearly $w_{q}\left(q^{m}\right)=\left(q^{m}-1\right) /(q-1)$, and if $n=\sum_{i=0}^{m-1} b_{i} q^{i}$ with $0 \leq b_{i} \leq q-1$ then

$$
w_{q}(n)=\sum_{i=0}^{m-1} b_{i} w_{q}\left(q^{i}\right) .
$$

It is well known (cf. [3, Prop. 1.3]) that

$$
\mathfrak{a}_{n}=\pi^{-w_{q}(n)} R .
$$

We give two examples of Mahler bases. To construct the first, let $\mathcal{T}$ be the set of Teichmuller representatives for $\kappa$ in $R$ (consisting of the roots of unity of order $q-1$ and the element 0 ). Let

$$
\mathcal{R}_{m}=\left\{\sum_{i=0}^{m-1} a_{i} \pi^{i} \mid a_{i} \in \mathcal{T}\right\}
$$

$\left(\mathcal{R}_{0}=\{0\}\right)$ and $\mathcal{R}=\bigcup \mathcal{R}_{m}$. There are $q^{m}$ elements in $\mathcal{R}_{m}$, constituting representatives for $R / \pi^{m} R$. Let

$$
g_{q^{m}}(x)=\pi^{-\left(q^{m}-1\right) /(q-1)} \prod_{r \in \mathcal{R}_{m}}(x-r)
$$

and if $n=\sum_{i=0}^{m-1} b_{i} q^{i}$ with $0 \leq b_{i} \leq q-1$ put

$$
g_{n}=\prod_{i=0}^{m-1} g_{q^{i}}^{b_{i}} .
$$

It is easy to check that this is an integral-valued polynomial of degree $n$, and since its leading coefficient generates $\mathfrak{a}_{n}$, the $g_{n}$ form a Mahler basis. This example appears already in [2]. 
For the second example we refer to [11]. Let $F$ be a Lubin-Tate formal group law over $R$ associated with the prime $\pi$ [5]. We denote by $t$ its parameter. The ring $R$ acts as endomorphisms of $F$, and we denote by

$$
[x](t)=x t+\cdots+c_{n}(x) t^{n}+\cdots
$$

the endomorphism corresponding to $x \in R$, uniquely determined by the condition $[x]^{\prime}(0)=x$. As a function of $x$, the coefficient $c_{n}$ is a polynomial of degree at most $n$ over $K$. This is seen by using the equation $[x] \circ[\pi]=[\pi] \circ[x]$ to deduce a recursive relation for the $c_{n}$. Since $c_{n}(x) \in R$ for $x \in R$ these polynomials belong to $\operatorname{Int}(R)$. In [11] it was shown that the $c_{q^{m}}$, for $m \geq 0$, generate $\operatorname{Int}(R)$ as a ring over $R$. It was shown there that $\operatorname{deg}\left(c_{q^{m}}\right)=q^{m}$, that the leading coefficient $\lambda_{q^{m}}$ of $c_{q^{m}}$ has valuation $v\left(\lambda_{q^{m}}\right)=-w_{q}\left(q^{m}\right)$, and that $\pi^{w_{q}\left(q^{m}\right)} c_{q^{m}}$ has integral coefficients.

It follows that if $n=\sum_{i=0}^{m-1} b_{i} q^{i}$ with $0 \leq b_{i} \leq q-1$ as above, then the polynomial

$$
f_{n}=\prod_{i=0}^{m-1} c_{q^{i}}^{b_{i}}
$$

is of degree $n$ and its leading coefficient has valuation $-w_{q}(n)$. The $\left\{f_{n}\right\}$ form our second example of a Mahler basis.

Remark 1.1. It is not possible to replace the $f_{n}$ by the $c_{n}$ in general. In fact, for $n$ that are not of the form $q^{m}$ the $c_{n}$ need not be of degree $n$. As an example, let $F$ be the formal group law of the elliptic curve $y^{2}=x^{3}-x$ over $\mathbb{Z}_{p}[i]\left(p \equiv 3 \bmod 4, i^{2}=-1\right)$, with respect to the formal parameter $t=-x / y$. Then $c_{n}(x)=0$ unless $n \equiv 1 \bmod 4$. We call the formal group law $F$ neat if for every $n, \operatorname{deg} c_{n}(x)=n$ and its leading coefficient $\lambda_{n}$ has valuation $v\left(\lambda_{n}\right)=-w_{q}(n)$. For neat Lubin Tate formal group laws, the $\left\{c_{n}\right\}$ also form a Mahler basis. It can be shown that neat formal group laws exist. In a certain sense, they are generic.

1.3. Mahler's theorem. Let $\left\{f_{n}\right\}$ be a Mahler basis. Let $\bar{f}_{n}: R \rightarrow \kappa$ be the function obtained from $f_{n}$ by reduction modulo $\pi$.

\section{Theorem 1.2.}

(i) The $\bar{f}_{n}(n \geq 0)$ form a basis for the space $C(R, \kappa)$ of continuous (i.e. locally constant) $\kappa$-valued functions on $R$.

(ii) Moreover, $\bar{f}_{0}, \ldots, \bar{f}_{q^{m}-1}$ form a basis for the subspace $C\left(R / \pi^{m} R, \kappa\right)$ of $\kappa$-valued functions that are constant on cosets of $\pi^{m} R$.

(iii) Every function in $C(R, K)$ has a unique uniformly convergent expansion

$$
f=\sum_{n=0}^{\infty} a_{n} f_{n}
$$


where $a_{n} \rightarrow 0$, and $|f|_{\text {sup }}=\max \left|a_{n}\right|$. Conversely, every such series converges uniformly and represents a continuous function.

We call $a_{n}$ the $n$th Mahler coefficient of $f$ (with respect to the particular basis).

Proof. Part (iii) follows from (i) by a standard argument, and (i) is a consequence of (ii). It is clear that the $\bar{f}_{n}$ are linearly independent over $\kappa$, or otherwise the $f_{n}$ would not span $\operatorname{Int}(R)$ over $R$. A dimension count therefore reduces the proof of the theorem to the verification that $\bar{f}_{n}$, for $n<q^{m}$, is constant on cosets of $\pi^{m} R$. Since the validity of this statement is independent of the particular Mahler basis at hand (if $g_{n}$ is another Mahler basis, each $g_{n}$ is an $R$-linear combination of $\left.f_{0}, \ldots, f_{n}\right)$ we may choose to work with the $f_{n}^{\prime} s$ from the second example. It is enough to prove that $\bar{c}_{n}$, for $n<q^{m}$, is constant on cosets of $\pi^{m} R$. This, in turn, follows from the following lemma.

Lemma 1.3. Let $y \in \pi^{m} R$. Then

$$
[x+y](t) \equiv[x](t) \bmod \left(\pi, t^{q^{m}}\right) .
$$

Proof. Let $\{x\}(t)=[x](t) \bmod \pi \in \kappa[[t]]$. Then

$$
\{x+y\}(t)=\bar{F}(\{x\}(t),\{y\}(t))
$$

where $\bar{F}$ is the reduction modulo $\pi$ of $F$. But $\bar{F}$ is of $\pi$-height $h$, where $q=p^{h}$, so $\{\pi\}=u t^{q}+$ (higher terms), and the lemma follows.

We remark that we could have concluded the proof of Theorem 1.2 with the aid of the basis (1.8) as well. For a generalization of the last lemma, see Proposition 2.4 below. As a corollary we get that the Weierstrass approximation theorem holds in $K$.

Corollary 1.4. (Kaplansky, [8, Theorem 43.3]) The polynomials are dense in $C(R, K)$.

Another corollary is the following. Let $\left\{f_{n}\right\}$ be a Mahler basis and let $f_{n}^{k}=f_{n} \bmod \pi^{k}$.

Corollary 1.5. The $R / \pi^{k} R$-module $C\left(R, R / \pi^{k} R\right)$ of locally constant $R / \pi^{k} R$-valued functions on $R$ is free, and $\left\{f_{n}^{k}\right\}$ is a basis for it.

\section{Mahler bases and the wavelet basis}

2.1. The basis of wavelets and the space of locally constant functions. With $\mathcal{T}$ and $\mathcal{R}$ defined as in $\S 1.2$, let the length of $r \in \mathcal{R}, l(r)=m$ if $r \in \mathcal{R}_{m}^{\prime}=\mathcal{R}_{m}-\mathcal{R}_{m-1}$. The basis of wavelets ${ }^{1}$ is the orthonormal basis

\footnotetext{
${ }^{1}$ The terminology is due to Colmez, see [4].
} 
of $C(R, K)$ given by $\left\{\chi_{r} \mid r \in \mathcal{R}\right\}$ where

$$
\chi_{r}=\text { the characteristic function of the disk } D_{r}=r+\pi^{l(r)} R \text {. }
$$

The locally constant (or step) functions $S(R, K)$ are precisely those admitting a finite expansion in terms of the $\chi_{r}$. The largest $l(r)$, for $\chi_{r}$ appearing in the expansion of $f \in S(R, K)$, is the smallest $l \geq 0$ such that $f$ is constant on cosets of $\pi^{l} R$. We call it the level of $f$, and denote it by $l(f)$. Thus $l\left(\chi_{r}\right)=l(r)$.

The last observation holds in fact with $K$ replaced by any commutative ring $L$.

Lemma 2.1. For any commutative ring $L$, the functions $\chi_{r}$ form an $L$ basis for the free module $S(R, L)$ of $L$-valued locally constant functions on R. Moreover, $\left\{\chi_{r} ; l(r) \leq l\right\}$ is a basis for the subspace $S\left(R / \pi^{l} R, L\right)$ of locally constant functions which are constant on cosets of $\pi^{l} R$.

2.2. The modulo- $k$ level and the level- $l$ modulus. Let $k \in \mathbb{Z}$. The $\bmod -k$-level $l_{k}(f)$ of $f \in C(R, K)$ is the smallest $l$ such that $f \bmod \pi^{k}$ is constant on cosets of $\pi^{l} R$. Let $l \geq 0$. The level-l-modulus $k_{l}(f)$ of $f$ is the maximal $k \in \mathbb{Z}$ such that $f \bmod \pi^{k}$ is constant on such cosets. If $f$ is locally constant and $l(f) \leq l$, then we put $k_{l}(f)=\infty$. If $f$ is not locally constant, then all $k_{l}(f)$ are finite. We record the definitions of the level-l-modulus and the mod- $k$-level of a function $f \in C(R, K)$ side by side:

$$
\begin{aligned}
& k_{l}(f)=\max \left\{k \leq \infty|| x-x^{\prime}|\leq| \pi^{l}|\Rightarrow| f(x)-f\left(x^{\prime}\right)|\leq| \pi^{k} \mid\right\} \\
& l_{k}(f)=\min \left\{0 \leq l|| x-x^{\prime}|\leq| \pi^{l}|\Rightarrow| f(x)-f\left(x^{\prime}\right)|\leq| \pi^{k} \mid\right\} .
\end{aligned}
$$

Lemma 2.2. If $f \in C(R, K)$ and

$$
f=\sum b_{r} \chi_{r}
$$

is the wavelet expansion of $f$, then

$$
l_{k}(f)=\max \left\{l(r)|| b_{r}|>| \pi^{k} \mid\right\} .
$$

Proof. Since $l_{k}(f)=l_{k+1}(\pi f)$ we may assume that $f$ is $R$-valued. Passing to $f \bmod \pi^{k}$ we see that the lemma is equivalent to the assertion that the level of an $f \in S\left(R, R / \pi^{k} R\right)$ is the maximal $l(r)$ for $r$ such that $\chi_{r}$ appears in the support of $f$. This follows from the previous lemma, with $L=R / \pi^{k} R$.

Lemma 2.3. We have the following inequalities

$$
\begin{aligned}
& k_{l}(f+g) \geq \min \left\{k_{l}(f), k_{l}(g)\right\} \\
& l_{k}(f+g) \leq \max \left\{l_{k}(f), l_{k}(g)\right\} .
\end{aligned}
$$

Equality holds if $k_{l}(f) \neq k_{l}(g)$ (resp. $l_{k}(f) \neq l_{k}(g)$ ). 
2.3. The relation between the Mahler coefficients and the wavelet coefficients. We turn to examine the expansion of a Mahler basis in the basis of wavelets. Lemma 1.3 can be rephrased as saying that $l_{1}\left(f_{n}\right) \leq m$ as long as $n<q^{m}$. We now generalize it.

Proposition 2.4. Let $\left\{f_{n}\right\}$ be a Mahler basis. Then $l_{k}\left(f_{n}\right) \leq m$ if $n<$ $q^{m+1-k}$.

Proof. If $\left\{g_{n}\right\}$ is another Mahler basis then $l_{k}\left(g_{n}\right) \leq \max \left\{l_{k}\left(f_{0}\right), \ldots, l_{k}\left(f_{n}\right)\right\}$. We may therefore prove the proposition for a particular Mahler basis, and once again we choose to work with the second example from Section 1.2. Let $I_{l}$ be the ideal of $R[[t]]$ generated by $\pi^{l} t, \pi^{l-1} t^{q}, \ldots, \pi t^{q^{l-1}}, t^{q^{l}}$. If $h \in I_{l}$ then $h \circ[\pi] \in I_{l+1}$. This follows from the fact that $[\pi] \in I_{1}$. By induction, if $y \in \pi^{l} R$ then $[y] \in I_{l}$, hence $[x+y]=F([x],[y]) \equiv[x] \bmod I_{l}$. It follows that the coefficients $c_{n}(x)$ and $c_{n}(x+y)$ of $t^{n}$ are congruent modulo $\pi^{k}$ if $n<q^{l+1-k}$ and $|y| \leq\left|\pi^{l}\right|$. This proves the proposition for the $c_{n}$, and from there it follows for the $f_{n}$ as well, as they are products of $c_{n}$ with smaller or equal indices.

Proposition 2.5. Let

$$
f_{n}=\sum b_{n, r} \chi_{r}
$$

be the wavelet expansion of the Mahler basis. If $n<q^{m}$, then $\left|b_{n, r}\right| \leq$ $\left|\pi^{l(r)-m}\right|$. Equivalently, if $n<q^{m}$ then $f_{n}^{k} \in C\left(R / \pi^{m+k-1} R, R / \pi^{k} R\right)$.

Proof. If $n<q^{m}$ then by the previous proposition $l_{k}\left(f_{n}\right) \leq m+k-1$. By Lemma 1.3, this means that if $l(r) \geq m+k$ then $\left|b_{n, r}\right| \leq\left|\pi^{k}\right|$.

Corollary 2.6. Let

$$
\chi_{r}=\sum a_{r, n} f_{n}
$$

be the Mahler expansion of $\chi_{r}$. If $l(r) \leq m$, then $\left|a_{r, n}\right| \leq\left|\pi^{k}\right|$ for every $n \geq q^{k+m-1}$. Equivalently, the $R$-module spanned by $f_{n}^{k}$ with $n<q^{k+m-1}$ contains $C\left(R / \pi^{m} R, R / \pi^{k} R\right)$.

Proof. Invert the matrix $\left(b_{n, r}\right)$.

The proposition and the corollary are the analogue of Proposition 1.21 of [4]. If we let $l(n)$ (the length of $n$ in its expansion in base $q$ ) stand for the smallest $m$ such that $n<q^{m}$ then they are also equivalent to the inequalities

$$
\begin{aligned}
& v\left(a_{r, n}\right) \geq l(n)-l(r) \\
& v\left(b_{n, r}\right) \geq l(r)-l(n) .
\end{aligned}
$$

They also yield the following:

$$
C\left(R / \pi^{m} R, R / \pi^{k} R\right) \subset\left\langle f_{0}^{k}, f_{1}^{k}, \ldots, f_{q^{m+k-1}-1}^{k}\right\rangle \subset C\left(R / \pi^{m+2 k-2} R, R / \pi^{k} R\right) .
$$


If $k=1$ these inclusions are equalities, in general they are not. The subspace $\left\langle f_{0}^{k}, f_{1}^{k}, \ldots, f_{n}^{k}\right\rangle$ is nevertheless independent of the particular Mahler basis. Can one describe it?

\section{The Lipschitz condition}

The results of this section can probably be deduced also from [2]. A function $f \in C(R, K)$ is Lipschitz (of order 1 ) if there is a positive constant $A$ such that

$$
\left|f(x)-f\left(x^{\prime}\right)\right| \leq A\left|x-x^{\prime}\right|
$$

We denote the subspace of Lipschitz functions by $\operatorname{Lip}(R)$. Since it contains the locally constant functions, it is dense in $C(R, K)$.

Lemma 3.1. The following are equivalent

(i) $f \in C(R, K)$ is Lipschitz.

(ii) $k_{l}(f)-l$ (for $l \geq 0$ ) is bounded below.

(iii) $l_{k}(f)-k$ (for $\left.k \geq 0\right)$ is bounded above.

Proof. The equivalence of (i) and (ii) is clear. As to the equivalence of (ii) and (iii) note that if $k_{l}(f) \geq l-K$ for all $l \geq 0$, then $\left|x-x^{\prime}\right| \leq\left|\pi^{l}\right| \Rightarrow$ $\left|f(x)-f\left(x^{\prime}\right)\right| \leq\left|\pi^{l-K}\right|$, so for $k \geq-K$ we have $l_{k}(f) \leq k+K$. The converse is equally easy.

Corollary 3.2. Let $b_{r}(f)$ (for $r \in \mathcal{R}$ ) be the wavelet coefficients of $f \in$ $C(R, K)$. Then $f \in \operatorname{Lip}(R)$ if and only if

$$
\omega(f)=\inf _{r}\left\{v\left(b_{r}(f)\right)-l(r)\right\}>-\infty .
$$

The function $|f|_{\text {Lip }}=\left|\pi^{\omega(f)}\right|$ is a Banach norm on Lip $(R)$.

Proof. By Lemma 2.2, $l_{k}(f)-k \leq K$ for all $k \geq-K$ if and only if $l(r)>$ $k+K \Rightarrow v\left(b_{r}(f)\right) \geq k$ for all such $k$ and every $r$, i.e., if and only if $v\left(b_{r}(f)\right) \geq l(r)-K-1$ for every $r$. By the previous lemma, $f$ is Lipschitz if and only if $\omega(f)>-\infty$. Now clearly $\omega(f)$ is finite for $f \neq 0$, since at least one $b_{r}(f) \neq 0, \omega(c f)=v(c)+\omega(f)$, and $\omega(f+g) \geq \min \{\omega(f), \omega(g)\}$, which means that $|\cdot|_{\text {Lip }}$ is a non-archimedean absolute value. The map $f \mapsto\left(\pi^{-l(r)} b_{r}(f)\right)_{r \in \mathcal{R}}$ is a norm-preserving isomorphism of $\operatorname{Lip}(R)$ with the Banach space $l^{\infty}(\mathcal{R})$ of all bounded functions on $\mathcal{R}$, hence $\operatorname{Lip}(R)$ is complete.

For example, $\left|\chi_{r}\right|_{\text {Lip }}=\left|\pi^{-l(r)}\right|$, and if $\left\{f_{n}\right\}$ is a Mahler basis, $\left|f_{n}\right|_{\text {Lip }}=$ $\left|\pi^{-l(n)}\right|$ (recall that $l(n)$ is the least $m \geq 0$ such that $n<q^{m}$ ). In fact, we have the following characterization of the Lipschitz condition in terms of the Mahler coefficients of $f$. 
Proposition 3.3. Let $\left\{f_{n}\right\}$ be a Mahler basis. A function $f=\sum a_{n}(f) f_{n} \in$ $C(R, K)$ is in Lip $(R)$ if and only if

$$
\omega^{\prime}(f)=\inf _{n}\left\{v\left(a_{n}(f)\right)-l(n)\right\}>-\infty
$$

and $\omega^{\prime}(f)=\omega(f)$.

Proof. Since

$$
a_{n}(f)=\sum_{r} b_{r}(f) a_{r, n}
$$

we have

$$
\begin{aligned}
v\left(a_{n}(f)\right)-l(n) & \geq \inf _{r}\left\{v\left(b_{r}(f)\right)+v\left(a_{r, n}\right)-l(n)\right\} \\
& \geq \inf _{r}\left\{v\left(b_{r}(f)\right)-l(r)\right\}=\omega(f)
\end{aligned}
$$

in view of the inequality $v\left(a_{r, n}\right) \geq l(n)-l(r)$. This shows that if $f \in$ $\operatorname{Lip}(R)$ then $\omega^{\prime}(f) \geq \omega(f)>-\infty$. In a completely analogous way, using the inequality $v\left(b_{n, r}\right) \geq l(r)-l(n)$ one proves that if $\omega^{\prime}(f)>-\infty$ then $f \in \operatorname{Lip}(R)$ and $\omega(f) \geq \omega^{\prime}(f)$.

When $R=\mathbb{Z}_{p}$ this proposition amounts to Theorem 53.4 in [8]. Note that $l(n) \approx \log _{q} n$.

\section{Locally analytic functions and Amice's theorem}

4.1. The spaces $\boldsymbol{L}_{\boldsymbol{h}}(\boldsymbol{R}, \boldsymbol{K})$. Let $h \geq 0$ be an integer. The space $L_{h}(R, K)$ of locally analytic functions of level $h$ is the subspace of $C(R, K)$ consisting of functions which are (rigid) analytic on every disk of radius $\left|\pi^{h}\right|$ with center in $R$. Such a function extends in a unique way to a rigid analytic function on

$$
\begin{aligned}
R[h] & =\left\{x \in \mathcal{K}|d(x, R) \leq| \pi^{h} \mid\right\} \\
& =\bigcup_{r \in \mathcal{R}_{h}} D\left(r,|\pi|^{h}\right)
\end{aligned}
$$

and

$$
|f|_{L_{h}}=\sup _{x \in R[h]}|f(x)|
$$

is a Banach norm on $L_{h}(R, K)$. Here we denote by $\mathcal{K}$ the completion of a fixed separable closure of $K$, by $d(x, R)$ the distance from $x$ to the compact set $R$, and by $D(a, \rho)$ the affinoid disk in $\mathcal{K}$ with center $a$ and radius $\rho$. The natural inclusions $L_{h}(R, K) \subset L_{h+1}(R, K)$ are compact (bounded subsets in $L_{h}(R, K)$ have compact closure in $\left.L_{h+1}(R, K)\right)$ and we endow the space

$$
L(R, K)=\bigcup L_{h}(R, K)
$$

of locally analytic functions with the inductive limit locally convex topology. See [9] for nonarchimedean functional analysis. 
4.2. Orthonormal bases in $\boldsymbol{L}_{h}(\boldsymbol{R}, \boldsymbol{K})$. Consider the polynomials $g_{n}$ defined in (1.8). Let

$$
\begin{aligned}
g_{q^{m}, h} & =g_{q^{m}} & & \text { if } m<h \\
& =\pi^{\left(q^{m-h}-1\right) /(q-1)} g_{q^{m}} & & \text { if } h \leq m,
\end{aligned}
$$

and

$$
g_{n, h}=\prod_{i=0}^{m-1} g_{q^{i}, h}^{b_{i}}
$$

if $n=\sum_{i=0}^{m-1} b_{i} q^{i}$ with $0 \leq b_{i} \leq q-1$.

Lemma 4.1. Fix $s \in \mathcal{R}_{h}$ and write

$$
g_{q^{m}, h}\left(s+\pi^{h} u\right)=\sum_{k=0}^{q^{m}} a_{k} u^{k} .
$$

(i) Assume $m<h$. Then $\left|a_{k}\right| \leq 1,\left|a_{k}\right|<1$ for $k>0$, and $\left|a_{0}\right|=1$ if and only if in the expansion

$$
s=s_{0}+s_{1} \pi+\cdots+s_{h-1} \pi^{h-1}
$$

$\left(s_{i} \in \mathcal{T}\right)$ the mth digit $s_{m} \neq 0$.

(ii) Assume $h \leq m$. Then $\left|a_{k}\right| \leq 1,\left|a_{q^{m-h}}\right|=1$ and $\left|a_{k}\right|<1$ for $q^{m-h}<k \leq q^{m}$.

Proof. Fix $s \in \mathcal{R}_{h}$ and consider the polynomial

$$
\Gamma(u)=\prod_{r \in \mathcal{R}_{m}}\left(s+\pi^{h} u-r\right) .
$$

(i) Assume $m<h$. As long as $r \neq s \bmod \pi^{m},\left|\pi^{h}\right|<|s-r|$. For the unique $r \equiv s \bmod \pi^{m}$, still $\left|\pi^{h}\right|<|s-r|=\left|\pi^{m}\right|$ if we assume that $s_{m} \neq 0$. An easy computation gives then

$$
\begin{aligned}
\operatorname{ord}_{\pi} \prod_{r \in \mathcal{R}_{m}}(s-r) & =\sum_{k=0}^{m-1} k\left(q^{m-k}-q^{m-k-1}\right)+m \\
& =\left(q^{m}-1\right) /(q-1),
\end{aligned}
$$

so, under the assumption that $s_{m} \neq 0,\left|g_{q^{m}, h}\left(s+\pi^{h} u\right)\right|=1$ for $|u| \leq 1$, and part (i) follows. If $s_{m}=0$, then the same computation shows that all the coefficients of $\Gamma(u)$ are divisible by $\pi^{\left(q^{m}-1\right) /(q-1)+1}$, so all the coefficients of $g_{q^{m}, h}$ satisfy $\left|a_{k}\right|<1$.

(ii) When $h \leq m$ we break $\Gamma(u)$ into the product over $r \neq s \bmod \pi^{h}$ and the product over $r \equiv s \bmod \pi^{h}$, where in the latter we use the substitution 
$r=s+\pi^{h} t, t \in \mathcal{R}_{m-h}$. We get

$$
\Gamma(u)=\prod_{r \in \mathcal{R}_{m}, r \neq s}(s-r)\left(1+\frac{\pi^{h} u}{s-r}\right) \cdot \prod_{t \in \mathcal{R}_{m-h}} \pi^{h}(u-t) .
$$

Note that in the first product $\left|\pi^{h}\right|<|s-r|$. Since

$$
\begin{aligned}
\operatorname{ord}_{\pi} & \prod_{r \in \mathcal{R}_{m}, r \neq s}(s-r) \cdot \pi^{h q^{m-h}} \bmod \pi^{h} \\
= & \sum_{k=0}^{h-1} k\left(q^{m-k}-q^{m-k-1}\right)+h q^{m-h} \\
& =\left(q^{m}-q^{m-h}\right) /(q-1),
\end{aligned}
$$

$g_{q^{m}, h}\left(s+\pi^{h} u\right)$ becomes, in the region $|u| \leq 1$, the product of a polynomial with constant absolute value 1 , and the polynomial

$$
\prod_{t \in \mathcal{R}_{m-h}}(u-t)
$$

so part (ii) follows.

The following was proved for $R=\mathbb{Z}_{p}$ by Amice. We follow Colmez's survey paper [4], Théorème I.4.7.

Proposition 4.2. The $g_{n, h}$ form an orthonormal basis for $L_{h}(R, K)$.

Proof. For

$$
n=\sum_{i=0}^{m-1} b_{i} q^{i}
$$

$\left(0 \leq b_{i} \leq q-1\right)$ put

$$
\tilde{n}=\sum_{i=h}^{m-1} b_{i} q^{i-h}=\left\lfloor\frac{n}{q^{h}}\right\rfloor .
$$

The previous lemma implies that if we write

$$
g_{n, h}\left(s+\pi^{h} u\right)=\sum_{k=0}^{n} a_{k} u^{k},
$$

then all $\left|a_{k}\right| \leq 1,\left|a_{k}\right|<1$ for $\tilde{n}<k$, and if none of the "digits" $s_{i}$ of $s$ vanishes, $\left|a_{\tilde{n}}\right|=1$. Hence

$$
\left|g_{n, h}\right|_{L_{h}}=1 \text {. }
$$

Let $m \geq h$ and consider the space $\mathcal{W}_{m-h}$ of all the functions from $R[h]$ to $\mathcal{O}_{\mathcal{K}} / \pi \mathcal{O}_{\mathcal{K}}$, which are the reduction of a polynomial of degree $<q^{m-h}$ on each $\pi^{h}$-residue disk in $R[h]$ separately. This is a free $\mathcal{O}_{\mathcal{K}} / \pi \mathcal{O}_{\mathcal{K}}$-module of rank $q^{m}$. By the lemma, the functions $\bar{g}_{n, h}$ obtained from $g_{n, h}$ upon 
reduction modulo $\pi$ belong there, if $n<q^{m}$. Among them, the functions $\bar{g}_{n}=\bar{g}_{n, h}$ for $n<q^{h}$ are constant on each residue disk, and constitute a basis for $\mathcal{W}_{0}$. This follows from part (ii) of Theorem 1.2. On the other hand the functions

$$
\prod_{i=h}^{m-1} \bar{g}_{q^{i}, h}^{b_{i}}
$$

are linearly independent over $\mathcal{O}_{\mathcal{K}} / \pi \mathcal{O}_{\mathcal{K}}$ on each $\pi^{h}$-residue disk in $R[h]$, since they are given there by monic polynomials of degrees $\sum_{i=h}^{m-1} b_{i} q^{i-h}$, and these degrees are distinct. We deduce that $\left\{\bar{g}_{n, h} ; 0 \leq n<q^{m}\right\}$ is a basis for the $q^{m}$-dimensional space $\mathcal{W}_{m-h}$. The reduction modulo $\pi$ of the unit ball of $L_{h}(R, K)$ is the union of all the $\mathcal{W}_{d}$ 's, and $\left\{\bar{g}_{n, h}\right\}$ form an algebraic basis for it. By a standard argument it follows that the $g_{n, h}$ form an orthonormal basis for the space $L_{h}(R, K)$.

4.3. Amice's theorem. This theorem characterizes functions in $L(R, K)$ in terms of the asymptotic decay of their Mahler coefficients. When $R=\mathbb{Z}_{p}$, it was proved in [1]. View also [4], Corollaire I.4.8.

Theorem 4.3. Let $\left\{f_{n}\right\}$ be a Mahler basis, and $f=\sum a_{n} f_{n} \in C(R, K)$. Then $f \in L(R, K)$ if and only if there exists an $\varepsilon>0$ such that $v\left(a_{n}\right)-\varepsilon n \rightarrow$ $\infty$ as $n \rightarrow \infty$.

Proof. The criterion being independent of the particular Mahler basis, we may work with the $g_{n}$ of (1.8). Recall that $f \in L(R, K)$ if and only if $f \in L_{h}(R, K)$ for some $h$, and by the last proposition, $f \in L_{h}(R, K)$ if and only if

$$
f=\sum a_{n, h} g_{n, h}
$$

where $a_{n, h} \rightarrow 0$. The relation between $g_{n, h}$ and $g_{n}$ yields $a_{n, h}=\pi^{-\omega_{n, h}} a_{n}$ where

$$
\omega_{n, h}=\sum_{i=h}^{m-1} b_{i} \frac{q^{i-h}-1}{q-1} .
$$

As usual, the $b_{i}$ are the digits of $n$ in base $q$. But

$$
\left\lfloor\frac{n}{q^{h+1}}\right\rfloor \leq(q-1) \omega_{n, h} \leq\left\lfloor\frac{n}{q^{h}}\right\rfloor
$$

so the existence of $h$ such that $f \in L_{h}(R, K)$ is equivalent to the existence of an $\varepsilon>0$ such that $v\left(a_{n}\right)-\varepsilon n \rightarrow \infty$ as $n \rightarrow \infty$. 


\section{Measures, distributions and the Iwasawa-Mahler-Amice transform}

5.1. Measures and the Iwasawa-Mahler transform. Let $C(R, K)^{\prime}$ be the Banach dual of $C(R, K)$, i.e. the space of bounded linear functionals on $C(R, K)$. It is well known that this space is identified (via integration) with the Iwasawa algebra of bounded measures on $R$. Let $\left\{f_{n}\right\}$ be a Mahler basis. The Iwasawa-Mahler transform of $\mu \in C(R, K)^{\prime}$ with respect to the given Mahler basis is the power series

$$
A_{\mu}(z)=\sum_{n=0}^{\infty}\left(\int_{R} f_{n}(x) d \mu(x)\right) z^{n} .
$$

For example, if $R=\mathbb{Z}_{p}$ and $f_{n}(x)=\left(\begin{array}{l}x \\ n\end{array}\right)$ is the classical Mahler basis, this takes the familiar form

$$
A_{\mu}(z)=\int_{\mathbb{Z}_{p}}(1+z)^{x} d \mu(x) .
$$

The following proposition is obvious, and encodes the fact that the dual of the Banach space $c_{0}$ is $l_{\infty}$.

Proposition 5.1 (Iwasawa, Mahler). The map $\mu \mapsto A_{\mu}$ is a norm preserving isomorphism of $K$-Banach spaces between $C(R, K)^{\prime}$ and $K \otimes R[[z]]$, which depends on the choice of a Mahler basis.

Since $K \otimes R[[z]]$ are just the $K$-rational bounded holomorphic functions in the open unit disk

$$
\Delta=\{z \in \mathcal{K} ;|z|<1\},
$$

and since the sup norm of such a function is the maximum of the absolute values of the coefficients in the power series representing it (the Gauss norm), we get the following corollary.

Corollary 5.2. The map $\mu \mapsto A_{\mu}$ is a norm preserving isomorphism of $K$-Banach spaces between $C(R, K)^{\prime}$ and the space $\mathcal{O}(\Delta)^{b}$ of $K$-rational bounded holomorphic functions on $\Delta$ (with the sup norm), which depends on the choice of a Mahler basis.

The subring $R[[z]]$ of holomorphic functions on $\Delta$ which are bounded by 1 is the classical Iwasawa algebra. The Iwasawa-Mahler transform of $\delta_{x}$, the Dirac measure at $x \in R$, will also be written $A_{\delta_{x}}=A_{x}$, so that

$$
A_{x}=\sum_{n=0}^{\infty} f_{n}(x) z^{n} .
$$

We emphasize that the isomorphism does not respect the ring structure (convolution of measures on one side and multiplication of bounded holomorphic functions on the other side) except in the case $R=\mathbb{Z}_{p}$ and the 
classical Iwasawa-Mahler transform. In this case $A_{\delta_{x}} A_{\delta_{y}}=A_{\delta_{x} * \delta_{y}}$, or

$$
A_{x} A_{y}=A_{x+y}
$$

because

$$
(1+z)^{x}(1+z)^{y}=(1+z)^{x+y} .
$$

It is easy to see that in general, there does not exist a Mahler basis that will make the transform a ring isomorphism. The fact that in the classical case the Iwasawa-Mahler transform respects the multiplicative structure is crucial in Colmez' work on the $p$-adic local Langlands correpondence.

There does not seem to be a satisfactory description of the $K$-Banach algebra $C(R, K)^{\prime}$ as a ring of holomorphic functions, except when $K=\mathbb{Q}_{p}$. However, when one replaces continuous distributions by locally analytic ones, the same question has been answered in the affirmative by Schneider and Teitelbaum [10] using Lubin-Tate theory. We explain the connection between our point of view, based on the notion of Mahler bases, and their results, below.

5.2. Locally analytic distributions and the Amice transform. We first generalize the Iwasawa-Mahler transform to locally analytic distributions, where it will be called the Amice transform. Recall that the space of locally analytic distributions is the topological dual $L(R, K)^{\prime}$ of $L(R, K)$.

5.2.1. Some rings of holomorphic functions. As above, let $\Delta$ be the open unit disk, viewed as a rigid analytic space over $K$. Let $\dot{\Delta}$ be its complement in $\mathbb{P}^{1}(\mathcal{K})$ :

$$
\dot{\Delta}=\{z \in \mathcal{K}|| z \mid \geq 1\} \cup\{\infty\} .
$$

For any $K$-rational rigid open set $U \subset \mathbb{P}^{1}(\mathcal{K})$ we denote by $\mathcal{O}(U)$ the ring of $K$-rational holomorphic (rigid analytic) functions on $U$, and by $\mathcal{O}(U)^{b}$ the subring of bounded ones. If $U=\bigcup U_{n}$ where each $U_{n}$ is an affinoid and $U_{n} \subset \subset U_{n+1}$ then we topologize $\mathcal{O}(U)$ as the inverse limit of the $K$-Banach algebras $\mathcal{O}\left(U_{n}\right)$, and as such it becomes a $K$-Fréchèt space. On $\mathcal{O}(U)^{b}$ we put the supremum norm, in which it is complete (Banach).

Consider the two rings

$$
\begin{aligned}
& \mathcal{O}(\dot{\Delta})_{0}=\left\{\sum_{n=0}^{\infty} a_{n} z^{-n-1} \mid a_{n} \in K, \quad v\left(a_{n}\right) \rightarrow \infty\right\} \supset \\
& \mathcal{O}(\dot{\Delta})_{0}^{\dagger}=\left\{\sum_{n=0}^{\infty} a_{n} z^{-n-1} \mid a_{n} \in K, \quad \exists \varepsilon>0, v\left(a_{n}\right)-\varepsilon n \rightarrow \infty\right\} .
\end{aligned}
$$

The first is the $K$-Banach algebra of functions which are holomorphic on $\dot{\Delta}$ and vanish at $\infty$. The second is its subring consisting of overconvergent 
functions, i.e. those which converge on

$$
\dot{\Delta}_{r}=\{z \in \mathcal{K} \| z \mid \geq r\} \cup\{\infty\}
$$

for some $r<1$. We topologize it as the direct limit of the $K$-Banach algebras $\mathcal{O}\left(\dot{\Delta}_{r}\right)_{0}$. It is then a space of compact type $[9]$.

The topological duals of these two spaces are, respectively,

$$
\begin{aligned}
\mathcal{O}(\Delta)^{b} & =\left\{\sum_{n=0}^{\infty} a_{n} z^{n} \mid a_{n} \in K \text { is bounded }\right\} \subset \\
\mathcal{O}(\Delta) & =\left\{\sum_{n=0}^{\infty} a_{n} z^{n} \mid a_{n} \in K ; \quad \forall \varepsilon>0, v\left(a_{n}\right)+\varepsilon n \rightarrow \infty\right\}
\end{aligned}
$$

with their Banach (resp. Fréchèt) topology. The duality pairing is

$$
\langle f(z), g(z)\rangle=\operatorname{res}(f(z) g(z) d z)
$$

where the residue res is the coefficient of $z^{-1} d z$.

We emphasize that although the second pair of spaces are reflexive [9], so $\mathcal{O}(\dot{\Delta})_{0}^{\dagger}$ is also the topological dual of $\mathcal{O}(\Delta)$, this is not true of the first pair. The space $\mathcal{O}(\Delta)^{b}$ is the dual of $\mathcal{O}(\dot{\Delta})_{0}$, but not vice versa, just as $l_{\infty}$ is the dual of $c_{0}$, but not the other way around.

5.2.2. Relation to Mahler expansions. Now fix a Mahler basis as before and use it to identify $f=\sum_{n=0}^{\infty} a_{n} f_{n} \in C(R, K)$ with $\sum_{n=0}^{\infty} a_{n} z^{-n-1} \in$ $\mathcal{O}(\dot{\Delta})_{0}$ as in Mahler's theorem. The locally analytic functions $L(R, K)$ are then identified with $\mathcal{O}(\Delta)_{0}^{\dagger}$. This is just a restatement of Amice's theorem on the asymptotic decay of Mahler coefficients of locally analytic functions.

Since $f_{n}(x) \in L(R, K)$, the Iwasawa-Mahler transform $\mu \mapsto A_{\mu}$ extends from $C(R, K)^{\prime}$, where it induces an isomorphism with $\mathcal{O}(\Delta)^{b}$, to $L(R, K)^{\prime}$. This extension, which we call the Amice transform, is an isomorphism between $L(R, K)^{\prime}$ and $\mathcal{O}(\Delta)$. This is clear from the discussion in the previous section. We summarize everything in the following theorem.

Theorem 5.3. Fix a Mahler basis $\left\{f_{n}\right\}$ as above. Then Mahler expansions and the Amice transform induce topological isomorphisms

$$
C(R, K) \simeq \mathcal{O}(\dot{\Delta})_{0}, \quad C(R, K)^{\prime} \simeq \mathcal{O}(\Delta)^{b}
$$

of K-Banach spaces (and their Banach duals) and

$$
L(R, K) \simeq \mathcal{O}(\dot{\Delta})_{0}^{\dagger}, \quad L(R, K)^{\prime} \simeq \mathcal{O}(\Delta)
$$

of spaces of compact type (and their Fréchèt duals). The isomorphisms are compatible with duality. 
5.2.3. Some rings of p-adic Hodge theory. This subsection will not be needed in what follows, and we include it for convenience and reference only. In $p$-adic Hodge theory one considers the following spaces, which are closed under multiplication.

$$
\begin{aligned}
\mathcal{E} & =\left\{\sum_{n \in \mathbb{Z}} a_{n} z^{n} \mid a_{n} \text { bounded, } a_{n} \rightarrow 0 \text { as } n \rightarrow-\infty\right\} \\
& \simeq \mathcal{O}(\dot{\Delta})_{0} \oplus \mathcal{O}(\Delta)^{b} \simeq C(R, K) \oplus C(R, K)^{\prime} .
\end{aligned}
$$

This ring, introduced by Fontaine, is in fact a field, and the non-archimedean norm sup $\left|a_{n}\right|$ is a multiplicative Banach norm on it, i.e. $\mathcal{E}$ is $p$-adically complete. Note that power series in $\mathcal{E}$ need not converge anywhere ${ }^{2}$. The unit ball $\mathcal{O}_{\mathcal{E}}$ in $\mathcal{E}$ is a Cohen ring for the field $\kappa((z)$ ) (a complete discrete valuation ring with $\kappa((z))$ as a residue field). The Iwasawa algebra $K \otimes R[[z]] \simeq \mathcal{O}(\Delta)^{b}$ is sometimes denoted $\mathcal{E}^{+}$.

Similarly, we can put together the locally analytic functions and distributions to get

$$
\begin{aligned}
\mathcal{R} & =\left\{\begin{array}{c}
\sum_{n \in \mathbb{Z}} a_{n} z^{n} \mid \forall \varepsilon>0, v\left(a_{n}\right)+\varepsilon n \rightarrow \infty \text { as } n \rightarrow \infty \\
\exists \varepsilon>0, v\left(a_{n}\right)+\varepsilon n \rightarrow \infty \text { as } n \rightarrow-\infty
\end{array}\right\} \\
& \simeq \mathcal{O}(\dot{\Delta})_{0}^{\dagger} \oplus \mathcal{O}(\Delta)=\lim _{\rightarrow} \mathcal{O}\left(\Delta_{[r, 1)}\right) \simeq L(R, K) \oplus L(R, K)^{\prime} .
\end{aligned}
$$

Here $\Delta_{[r, 1)}=\{z|r \leq| z \mid<1\}$. This is the Robba ring ${ }^{3}$.

The "common part" of $\mathcal{E}$ and $\mathcal{R}$ is the bounded Robba ring

$$
\mathcal{E}^{\dagger}=\mathcal{O}(\dot{\Delta})_{0}^{\dagger} \oplus \mathcal{O}(\Delta)^{b}=\lim _{\rightarrow} \mathcal{O}\left(\Delta_{[r, 1)}\right)^{b} \simeq L(R, K) \oplus C(R, K)^{\prime} .
$$

The ring $\mathcal{E}$ is the $p$-adic completion of $\mathcal{E}^{\dagger}$.

We note that it is impossible to make a larger ring in which both $\mathcal{E}$ and $\mathcal{R}$ embed by taking the direct sum of $C(R, K)$ with $L(R, K)^{\prime}$. The collection of power series obtained in this way will not form a ring.

\section{Relation to $p$-adic Fourier theory}

From now on we assume that we are in the "mixed characteristic case"

$$
\operatorname{char}(K)=0 .
$$

\footnotetext{
${ }^{2}$ It is tempting to speculate that $\mathcal{E}$ is the ring of analytic functions on a "non-standard annulus" in a non-standard model of the $p$-adics.

${ }^{3}$ As we shall have no use for the Robba ring in this paper, no confusion should arise from denoting it by the same letter which was used to denote the set of representatives $\mathcal{R}$.
} 
6.1. Recall of the results of Schneider and Teitelbaum. As indicated above, the Mahler expansion, and dually, the Iwasawa-Mahler-Amice transform $\mu \mapsto A_{\mu}$, depend on the choice of a Mahler basis. In addition, the Amice transform is a norm preserving isomorphism of $K$-Banach spaces (for measures) or $K$-Fréchèt spaces (for locally analytic distributions), but does not respect the multiplicative structures given by convolution on $C(R, K)^{\prime}$ (resp. $\left.L(R, K)^{\prime}\right)$, and by multiplication in $\mathcal{O}(\Delta)^{b}$ (resp. $\mathcal{O}(\Delta)$ ), except in the classical case.

In a beautiful but somewhat overlooked paper [10], Schneider and Teitelbaum found that, for the locally analytic distributions, both problems can be fixed if we replace the unit disk $\Delta$ by a twisted version. This twisted version is a rigid analytic space $\tilde{\Delta}$ defined over $K$, which becomes isomorphic to $\Delta$ over $\mathcal{K}$, or, somewhat better, over a large complete subfield of $\mathcal{K}$ which will be described in due course. The main result of Schneider and Teitelbaum is the construction of a canonical $K$-algebra isomorphism

$$
\mu \mapsto \tilde{A}_{\mu}, \quad L(R, K)^{\prime} \simeq \mathcal{O}(\tilde{\Delta})
$$

An equivalent formulation is that over $\mathcal{K}$ there is an isomorphism of topological $\mathcal{K}$-algebras between $L(R, \mathcal{K})^{\prime}$ and the $\operatorname{ring} \mathcal{O}(\tilde{\Delta}, \mathcal{K})$ of $\mathcal{K}$-rational holomorphic functions on the unit disk. This isomorphism is Galois equivariant for the natural action on $L(R, \mathcal{K})^{\prime}$, and for a twisted Galois action on $\mathcal{O}(\tilde{\Delta}, \mathcal{K})$, so descends to an isomorphism between $L(R, K)^{\prime}$ and $\mathcal{O}(\tilde{\Delta})$.

The approach taken by Schneider and Teitelbaum is Fourier-analytic. They begin by analyzing the locally $K$-analytic characters of $R$. For any complete field $K \subset L \subset \mathcal{K}$, denote by $\mathfrak{X}(L)$ the group of locally $K$-analytic homomorphisms $\chi: R \rightarrow U_{1}(L)$, where $U_{1}(L)=1+\mathfrak{m}_{L}$ is the multiplicative group of principal units of $L$.

When $R=\mathbb{Z}_{p}$ every continuous character is locally analytic, so $\mathfrak{X}$ is represented by the open unit disk $\Delta$, the point $z \in \Delta(L)$ corresponding to the character $\chi_{z} \in \mathfrak{X}(L)$ given by

$$
\chi_{z}(x)=(1+z)^{x}=\sum_{n=0}^{\infty}\left(\begin{array}{l}
x \\
n
\end{array}\right) z^{n} .
$$

Viewed as a function of $x, \chi_{z}(x)$ is locally analytic and its Mahler coefficients are $z^{n}$. Viewed as a function of $z$ (for a fixed $x$ ), we see that $\chi_{z}(x)$ is holomorphic (rigid analytic) in $\Delta$. In this case $\tilde{A}_{\mu}=A_{\mu}$ is given by

$$
A_{\mu}(z)=\int_{\mathbb{Z}_{p}} \chi_{z}(x) d \mu(x)
$$

In the following sections we give an account of the paper [10]. 


\subsection{Lubin-Tate groups and the description of $\mathfrak{X}(\mathcal{K})$.}

6.2.1. Lubin-Tate groups and their Cartier duals. Recall that in Chapter 1 we introduced a Lubin-Tate formal group law $F$ associated to the uniformizer $\pi$ [5]. This formal group law is a certain power series $F \in R[[X, Y]]$, and the formal group $\widehat{\mathcal{G}}_{/ R}$ that it defines depends only on $\pi$, up to isomorphism. According to the well-known equivalence between formal groups and $p$-divisible groups [12], giving $\widehat{\mathcal{G}}$ is the same as giving a certain $p$-divisible group $\mathcal{G}$ over $R$. Its special fiber $\mathcal{G}_{/ \kappa}$ is connected, onedimensional and of $p$-height $\left[K: \mathbb{Q}_{p}\right]$. Moreover, $\mathcal{G}$ carries an action of $R$ as endomorphisms, so $\widehat{\mathcal{G}}$ becomes a formal $R$-module, and as such has $\pi$-height $\left[\kappa: \mathbb{F}_{p}\right]$.

The precise relation between $F$ and $\mathcal{G}$ is the following. The $p$-divisible group $\mathcal{G}$ is a direct system $\left(\mathcal{G}_{n}\right)$ of finite flat group schemes $\left(\mathcal{G}_{n}=\mathcal{G}\left[p^{n}\right]\right)$. Each $\mathcal{G}_{n}=\operatorname{Spec}\left(A_{n}\right)$ where $A_{n}$ is finite and flat over $R$, and is equipped with a co-multiplication making it into a Hopf algebra. In fact, $A_{n}=$ $R[[Z]] /\left(\left[p^{n}\right]_{F}\right)$. The assumptions imply that

$$
A=\lim _{\leftarrow} A_{n}=R[[Z]]
$$

and the co-multiplication in $A$ is given by the original power series $F$.

Let $\mathcal{G}^{\prime}$ be the dual $p$-divisible group. Recall that $\mathcal{G}^{\prime}{ }_{\kappa}$ is also of height $\left[K: \mathbb{Q}_{p}\right]$ but of dimension $\left[K: \mathbb{Q}_{p}\right]-1$. For any $R$-algebra $S$ one has

$$
\mathcal{G}_{n}^{\prime}(S)=\operatorname{Hom}\left(\mathcal{G}_{n / S}, \mu_{p^{n} / S}\right),
$$

where by Hom we mean homomorphisms of finite flat group schemes.

Let $K \subset L \subset \mathcal{K}$ be a complete field over which $\mathcal{G}^{\prime}$ is trivialized (it is here that we need char. $K=0$ ), and let $S$ be the ring of integers of $L$. Since $\mathcal{G}_{n / S}^{\prime} \rightarrow \operatorname{Spec}(S)$ is proper and separated, the valuative criterion for properness implies that

$$
\mathcal{G}_{n}^{\prime}(S) \rightarrow \mathcal{G}_{n}^{\prime}(L)
$$

is a bijection. The action of $R$ on $\mathcal{G}$ induces a dual action on $\mathcal{G}^{\prime}$, and $\mathcal{G}_{n}^{\prime}(L)$ becomes a cyclic $R$-module, noncanonically isomorphic to $R / p^{n} R$. The Galois action on $\mathcal{G}_{n}^{\prime}(L)$ is given by a character

$$
\tau_{n}^{\prime}: \operatorname{Gal}_{K} \rightarrow\left(R / p^{n} R\right)^{\times} .
$$

These characters fit together to give $\tau^{\prime}: G a l_{K} \rightarrow R^{\times}$. If $\tau: G a l_{K} \rightarrow R^{\times}$is the Lubin-Tate character associated with $\mathcal{G}$ then, by Cartier duality,

$$
\tau^{\prime}=\tau^{-1} \chi_{c y c}
$$

where $\chi_{c y c}: G a l_{K} \rightarrow \mathbb{Z}_{p}^{\times}$is the cyclotomic character.

The Tate module

$$
T^{\prime}=\lim _{\leftarrow} \mathcal{G}_{n}^{\prime}(L)
$$


(inverse limit under multiplication-by- $p$ maps) is free of rank 1 over $R$, and we let $t^{\prime}$ be a generator. Then

$$
\sigma\left(t^{\prime}\right)=\tau^{\prime}(\sigma) \cdot t^{\prime}
$$

for any $\sigma \in G a l_{K}$.

6.2.2. The period of $\mathcal{G}$ associated with $\boldsymbol{t}^{\prime}$. Let $\mu$ denote the $p$-divisible group of roots of unity. Any $t^{\prime} \in T^{\prime}$ determines a homomorphism of $p$ divisible groups from $\mathcal{G}_{/ S}$ to $\mu_{/ S}$, or, what is the same, a homomorphism $\phi_{t^{\prime}}$ of formal groups defined over $S$ from $\widehat{\mathcal{G}}$ to $\widehat{\mathbb{G}}_{m}$. In terms of the canonical parameter $X$ of $\widehat{\mathbb{G}}_{m}$, and the parameter $Z$ of $\widehat{\mathcal{G}}$ corresponing to $F$, it is given by a power series

$$
\phi_{t^{\prime}}^{*}(X)=\Phi_{t^{\prime}}=\Omega Z+\cdots \in S[[Z]] .
$$

We call $\Omega$ the period of the Lubin Tate group $\mathcal{G}$ associated with $t^{\prime}$ (and the choice of the parameter $Z$ ), and denote it by $\Omega_{t^{\prime}}$ when we wish to emphasize the dependence on $t^{\prime}$.

Recall that $v$ has been normalized so that $v(\pi)=1$. Let $v(p)=e$ be the absolute ramification index of $K$. Part (i) of the following proposition was proved in the appendix to [10] using results of Fontaine. We give here an elementary power-series proof.

\section{Proposition 6.1.}

(i) Assume that $t^{\prime}$ is a generator of $T^{\prime}$ as an $R$-module. Then

$$
v(\Omega)=\frac{e}{p-1}-\frac{1}{q-1} .
$$

(ii) For every $\sigma \in G l_{K}$,

$$
\Omega^{\sigma-1}=\tau^{\prime}(\sigma)
$$

(iii) For $r \in R$ let $r t^{\prime}=t^{\prime} \circ[r]$. Then

$$
\Omega_{r t^{\prime}}=r \Omega_{t^{\prime}}
$$

We need two lemmas.

Lemma 6.2. If $\Phi_{t^{\prime}}(\omega)=0$ for some $\omega \in \widehat{\mathcal{G}}(\mathcal{K})$, then $\omega$ is a torsion point of $\widehat{\mathcal{G}}$.

Proof. Since $\Phi_{t^{\prime}}$ is a group homomorphism, $\Phi_{t^{\prime}}([a] \omega)=0$ for every $a \in \mathbb{Z}_{p}^{\times}$. But all the $[a](\omega)$, for $a \in \mathbb{Z}_{p}^{\times}$, have the same valuation. As a power series which converges on the open unit disk can have only finitely many zeroes of a given valuation, $\omega$ must be a torsion point.

The second lemma is well known [7]. 
Lemma 6.3. Let

$$
f(Z)=b Z\left(1+b_{1} Z+b_{2} Z^{2}+\cdots\right)
$$

be a power series with coefficients in $\mathcal{K}$, and assume that it converges for $|Z| \leq 1$, and has no zeroes for $0<|Z|<1$. Then $\left|b_{i}\right| \leq 1, b_{i} \rightarrow 0$, and on the annulus $|Z|=1$, the function $f$ has only finitely many zeroes. If $|\zeta|=1$ then either $f$ has a zero in the residue disk $|Z-\zeta|<1$, in which case $|f(Z)|<|b|$ throughout that disk, or $|f(Z)|=|b|$ for every $Z$ satisfying $|Z-\zeta|<1$.

Proof. (i) Let $\rho \in \mathcal{K}$ be an element of valuation $v(\rho)=1 /(q-1)$ and consider

$$
f(Z)=\Phi_{t^{\prime}}(\rho Z)=\rho \Omega Z\left(1+b_{1} Z+b_{2} Z^{2}+\cdots\right),
$$

which clearly converges for $|Z| \leq 1$. Suppose that $f(\zeta)=0$, where $0<$ $|\zeta|<1$. Then $\Phi_{t^{\prime}}(\omega)=0$ for $\omega=\rho \zeta$, and by the first lemma $\omega$ must be a torsion point of $\widehat{\mathcal{G}}$. But the smallest non-zero torsion points of the Lubin-Tate group $\widehat{\mathcal{G}}$ are of valuation $1 /(q-1)$. This contradiction shows that $f$ does not have any zero in $0<|Z|<1$, so the assumptions of the second lemma are satisfied. This lemma tells us then that we may find the valuation of $\rho \Omega$ by looking at $|f(Z)|$ on any residue disk inside the annulus $|Z|=1$, where $f$ does not have any zero.

Look at the $q-1$ non-zero $\pi$-torsion points of $\mathcal{G}$, which lie on the annulus $v(Z)=1 /(q-1)$ and are mapped by $\Phi_{t^{\prime}}$ to the set

$$
\left\{\varepsilon \mid(1+\varepsilon)^{p}=1\right\} .
$$

We claim that at least one of them does not map to 0 . In fact, if they all map to 0 , the homomorphism $\phi_{t^{\prime}}$ would factor through $[\pi]$, and $t^{\prime}$ would not be a generator of $T^{\prime}$ as an $R$-module. Let $\omega=\rho \zeta$ be a $\pi$-torsion point of $\mathcal{G}$ which maps to $\varepsilon \neq 0$. Then $|\zeta|=1$ and $f(\zeta)=\Phi_{t^{\prime}}(\omega)=\varepsilon$. But $f$ does not have any zeroes in the residue disk $|Z-\zeta|<1$, because $\zeta$ is the only point in its residue disk for which $\rho \zeta$ is a torsion point of $\mathcal{G}$, and $\Phi_{t^{\prime}}$ can only vanish at torsion points. It follows that

$$
|\rho \Omega|=|f(\zeta)|=|\varepsilon| \text {. }
$$

As $v(\varepsilon)=e /(p-1)$ and $v(\rho)=1 /(q-1)$, part (i) follows.

(ii) Consider the homomorphism

$$
\left[\chi_{c y c}(\sigma)^{-1}\right]_{\mathbb{G}_{m}} \circ \phi_{t^{\prime}}^{\sigma} \circ[\tau(\sigma)]_{F}
$$

of $\mathcal{G}$ to $\mu$. It is easily checked that its effect on any element of $\mathcal{G}_{n}(\mathcal{K})$, for any $n$, is the same as that of $\phi_{t^{\prime}}$, hence they must coincide. Comparing the coefficient of $Z$ in the power series representing both homomorphisms, we get part (ii).

Part (iii) is obvious. 
6.2.3. The characters $\chi_{\boldsymbol{z}, t^{\prime}}(\boldsymbol{x})$. For any $Z \in \widehat{\mathcal{G}}(\mathcal{K})$, i.e. $Z \in \mathcal{K}$ with $|Z|<1$, viewed as a point on the Lubin-Tate formal group $\widehat{\mathcal{G}}$ via the parameter $Z$, and any $t^{\prime} \in T^{\prime}$, we let

$$
\left\langle t^{\prime}, Z\right\rangle=1+\Phi_{t^{\prime}}(Z)=1+\Omega Z+\cdots \in U_{1}(\mathcal{K}) .
$$

Using the logarithm $\log _{F}$ of the formal group law $F$ we can also write

$$
\left\langle t^{\prime}, Z\right\rangle=\exp \left(\Omega \log _{F}(Z)\right) .
$$

Note that this is a power series with coefficients in the ring of integers $S$.

For $x \in R$ and $z \in \widehat{\mathcal{G}}(\mathcal{K})$ let

$$
\chi_{z, t^{\prime}}(x)=\left\langle t^{\prime},[x](z)\right\rangle=1+\Phi_{t^{\prime}}([x](z)) .
$$

Since $\chi_{z, t^{\prime}}(x+y)=\chi_{z, t^{\prime}}(x) \cdot \chi_{z, t^{\prime}}(y)$, this is a locally analytic character, i.e.

$$
\chi_{z, t^{\prime}} \in \mathfrak{X}(\mathcal{K}) \subset L(R, \mathcal{K}) .
$$

If $r \in R$ we let $r t^{\prime}=t^{\prime} \circ[r]$. Then

$$
\chi_{z, r t^{\prime}}(x)=\chi_{z, t^{\prime}}(r x)=\chi_{[r](z), t^{\prime}}(x) .
$$

If we write

$$
[x](z)=\sum_{n=1}^{\infty} c_{n}(x) z^{n}
$$

where $c_{n} \in \operatorname{Int}_{n}(R)$ as in Chapter 1 , then

$$
\chi_{z, t^{\prime}}(x)=\sum_{n=0}^{\infty} C_{n, t^{\prime}}(x) z^{n}
$$

where $C_{n, t^{\prime}} \in \operatorname{Int}_{n}(R, S)$ ( $S$-valued polynomials on $R$ of degree at most $\left.n\right)$, $C_{0, t^{\prime}}=1$ and

$$
C_{n, t^{\prime}}(x) \equiv \Omega_{t^{\prime}} c_{n}(x) \quad \bmod \operatorname{Int}_{n-1}(R, S)
$$

In the notation of [10], $C_{n, t^{\prime}}(x)=P_{n}(\Omega x)$.

We conclude that for a fixed $x, \chi_{z, t^{\prime}}(x)$ is a rigid analytic function of $z$, and is in fact a homomorphism from $\widehat{\mathcal{G}}(\mathcal{K})$ to $U_{1}(\mathcal{K})$.

For a fixed $z, \chi_{z, t^{\prime}}(x)$ is a locally analytic function of $x$. Choosing a Mahler basis $\left\{f_{n}\right\}$ and expanding

$$
C_{n, t^{\prime}}(x)=\sum_{m=0}^{n} c_{n, m}^{t^{\prime}} f_{m}(x)
$$


$\left(c_{n, m}^{t^{\prime}} \in S\right)$ we get its Mahler expansion

$$
\begin{aligned}
\chi_{z, t^{\prime}}(x) & =\sum_{n=0}^{\infty} \sum_{m=0}^{n} c_{n, m}^{t^{\prime}} f_{m}(x) z^{n} \\
& =\sum_{m=0}^{\infty}\left(\sum_{n=m}^{\infty} c_{n, m}^{t^{\prime}} z^{n}\right) f_{m}(x) .
\end{aligned}
$$

Proposition 6.4 ([10, Prop. 3.1]). The map $\left(t^{\prime}, z\right) \mapsto \chi_{z, t^{\prime}}$ is a $\mathrm{Gal}_{K^{-}}$ equivariant isomorphism of groups

$$
T^{\prime} \otimes_{R} \widehat{\mathcal{G}}(\mathcal{K}) \simeq \mathfrak{X}(\mathcal{K}) .
$$

Proof. That this map is a Galois equivariant group homomorphism is clear. Let $t^{\prime}$ be a generator of $T^{\prime}$ as an $R$-module. If $\Phi_{t^{\prime}}([x](z))=0$ for all $x$, then as we have seen before, $z$ must be a torsion point, or else $\Phi_{t^{\prime}}(Z)$ would have infinitely many zeroes of the same valuation. But if $z$ is a primitive torsion point of level $\pi^{n}$, then $\phi_{t^{\prime}}$ would factor through $\left[\pi^{n}\right]$, contradicting the fact that $t^{\prime}$ is a generator of $T^{\prime}$, hence $z=0$. This shows the injectivity of the $\operatorname{map}\left(t^{\prime}, z\right) \mapsto \chi_{z, t^{\prime}}$. For the surjectivity we refer to [10] and the analysis of the diagram therein, which allows one to obtain that every $\chi \in \mathfrak{X}(\mathcal{K})$ is a $\chi_{z, t^{\prime}}$ as a consequence of [12, Prop. 11].

6.2.4. The twisted disk $\tilde{\boldsymbol{\Delta}}$. Let $\mathfrak{X}_{0}$ be the functor associating to any $L$ the group of $\mathbb{Q}_{p}$-locally-analytic homomorphisms from $R$ to $U_{1}(L)$. Let $d=\left[K: \mathbb{Q}_{p}\right]$. Since $R \simeq \mathbb{Z}_{p}^{d}$ as a $\mathbb{Q}_{p}$-analytic group, fixing a basis $\left(e_{1}, \ldots, e_{d}\right)$ of $R$ over $\mathbb{Z}_{p}$ shows that $\mathfrak{X}_{0}$ is represented by $\Delta^{d}$, and determines $d$ coordinates on $\mathfrak{X}_{0}$ : the point $\left(z_{1}, \ldots, z_{d}\right) \in \Delta^{d}$ corresponds to the $\mathbb{Q}_{p}$-locallyanalytic character sending $e_{i} \mapsto 1+z_{i}$. It is easy to see that $\mathfrak{X}$ is a closed subgroup of $\mathfrak{X}_{0}$, hence a rigid analytic space in its own right. In fact, $\mathfrak{X}$ is a smooth rigid analytic curve defined over $K$. The power series expansion $\chi_{z, t^{\prime}}(x)=\sum_{n=0}^{\infty} C_{n, t^{\prime}}(x) z^{n}$ shows that in terms of the above coordinates the embedding

$$
T^{\prime} \otimes_{R} \widehat{\mathcal{G}}(\mathcal{K}) \simeq \mathfrak{X}(\mathcal{K}) \subset \mathfrak{X}_{0}(\mathcal{K})
$$

is given by

$$
t^{\prime} \otimes z \mapsto\left(\sum_{n=1}^{\infty} C_{n, t^{\prime}}\left(e_{i}\right) z^{n}\right)_{i=1}^{d} .
$$

This means that over $\mathcal{K}$, and once we have fixed a choice of a generator $t^{\prime}$ of $T^{\prime}$, the isomorphism $T^{\prime} \otimes_{R} \widehat{\mathcal{G}}(\mathcal{K}) \simeq \mathfrak{X}(\mathcal{K})$ is given by an injective rigid analytic morphism from $\Delta$ to $\Delta^{d}$, whose image on $\mathcal{K}$-points is precisely $\mathfrak{X}(\mathcal{K})$. One can probably deduce from here by general principles that such a morphism induces an isomorphism between $T^{\prime} \otimes_{R} \widehat{\mathcal{G}} \simeq \Delta$ and $\mathfrak{X}$ as rigid 
analytic groups over $\mathcal{K}$, without going into detailed computations. However, in [10, Theorem 3.6], the authors do better. They find two explicit, increasing and exhausting sequences of affinoid subdomains (in $T^{\prime} \otimes_{R} \widehat{\mathcal{G}}$ and $\mathfrak{X}$ respectively) that map isomorphically to each other. The precise knowledge of $v\left(\Omega_{t^{\prime}}\right)$ for a generator of $T^{\prime}$ becomes indispensible here. In any case, $T^{\prime} \otimes_{R} \widehat{\mathcal{G}} \simeq \Delta$ and $\mathfrak{X}$ are proved to be isomorphic as rigid analytic group varieties over $\mathcal{K}$. By Galois descent we conclude that $\mathfrak{X}$ is isomorphic over $K$ to the $K$-form $\tilde{\Delta}$ of the unit disk given by the (cohomology class of the) continuous 1-cocycle

$$
\tau^{\prime} \in H^{1}\left(\operatorname{Gal}_{K}, \operatorname{Aut}_{\mathcal{K}}(\Delta)\right) .
$$

For any complete $K \subset L \subset \mathcal{K}$,

$$
\tilde{\Delta}(L)=\left(T^{\prime} \otimes_{R} \widehat{\mathcal{G}}(\mathcal{K})\right)^{G a l_{L}} \simeq \mathfrak{X}(L) .
$$

In particular, $\tilde{\Delta}$ becomes isomorphic to the unit disk over the splitting field of $\mathcal{G}^{\prime}$.

\subsection{The isomorphism $L(R, K)^{\prime} \simeq \mathcal{O}(\tilde{\Delta})$.}

6.3.1. Definition of $\tilde{A}_{\boldsymbol{\mu}}$ and first properties. Let $\mu \in L(R, \mathcal{K})^{\prime}$ and define

$$
\tilde{A}_{\mu}\left(t^{\prime} \otimes z\right)=\int_{R} \chi_{z, t^{\prime}}(x) d \mu(x) .
$$

Substituting the power series expansion of $\chi_{z, t^{\prime}}(x)$ (in $z$ ) and integrating we see at once that for a fixed $t^{\prime}$,

$$
A_{\mu, t^{\prime}}(z)=\tilde{A}_{\mu}\left(t^{\prime} \otimes z\right)
$$

is a rigid analytic function of $z$ in the unit disk. From the corresponding properties of $\chi_{z, t^{\prime}}(x)$ we get

$$
A_{\mu, r t^{\prime}}(z)=A_{r \mu, t^{\prime}}(z)=A_{\mu, t^{\prime}}([r](z)) .
$$

Here the $R$-action on $\mu$ is the one that extends the action $r \delta_{x}=\delta_{r x}$.

In particular, if $\sigma \in \mathrm{Gal}_{K}$ then $\sigma\left(t^{\prime}\right)=\tau^{\prime}(\sigma) t^{\prime}$, so

$$
A_{\mu, \sigma\left(t^{\prime}\right)}(z)=A_{\tau^{\prime}(\sigma) \mu, t^{\prime}}(z)=A_{\mu, t^{\prime}}\left(\left[\tau^{\prime}(\sigma)\right](z)\right) .
$$

The map $\mu \mapsto \tilde{A}_{\mu}$ is an algebra homomorphism. It is enough to check it for Dirac measures $\delta_{x}$ and $\delta_{y}$, where it follows from

$$
\chi_{z, t^{\prime}}(x+y)=\chi_{z, t^{\prime}}(x) \chi_{z, t^{\prime}}(y) .
$$

It is also Galois-equivariant. It therefore descends to a homomorphism

$$
L(R, K)^{\prime} \rightarrow \mathcal{O}(\tilde{\Delta})
$$




\subsubsection{The main theorem of [10].}

Theorem 6.5. The map $\mu \mapsto \tilde{A}_{\mu}$ is an isomorphism of $K$-Fréch èt algebras between $L(R, K)^{\prime}$ and $\mathcal{O}(\tilde{\Delta})$.

Proof. The theorem is a consequence of all that has been done, and the general fact that the Fourier transform

$$
\begin{gathered}
L(R, \mathcal{K})^{\prime} \rightarrow \mathcal{O}(\mathfrak{X}(\mathcal{K})) \\
\mu \mapsto\left(\chi \mapsto \int_{R} \chi d \mu\right)
\end{gathered}
$$

is a Galois-equivariant isomorphism. This is [10, Theorem 2.3].

\section{References}

[1] Y. Amice, "Interpolation p-adique", Bull. Soc. Math. France 92 (1964), p. 117-180.

[2] D. BARsky, "Fonctions $k$-lipschitziennes sur un anneau local et polynômes à valeurs entières", Bull. Soc. Math. France 101 (1973), p. 397-411.

[3] J.-L. Chabert \& P.-J. CAhen, "Old problems and new questions around integer-valued polynomials and factorial sequences", in Multiplicative ideal theory in commutative algebra, Springer, New York, 2006, p. 89-108.

[4] P. Colmez, "Fonctions d'une variable p-adique", Astérisque (2010), no. 330, p. 13-59.

[5] J. Lubin \& J. TAte, "Formal complex multiplication in local fields", Ann. of Math. (2) 81 (1965), p. 380-387.

[6] K. Mahler, "An interpolation series for continuous functions of a p-adic variable", J. Reine Angew. Math. 199 (1958), p. 23-34.

[7] P. Roquette, Analytic theory of elliptic functions over local fields, Hamburger Mathematische Einzelschriften (N.F.), Heft 1, Vandenhoeck \& Ruprecht, Göttingen, 1970, 90 pages.

[8] W. H. Schikhof, Ultrametric calculus, Cambridge Studies in Advanced Mathematics, vol. 4, Cambridge University Press, Cambridge, 1984, An introduction to $p$-adic analysis, viii+306 pages.

[9] P. SchneIder, Nonarchimedean functional analysis, Springer Monographs in Mathematics, Springer-Verlag, Berlin, 2002, vi+156 pages.

[10] P. Schneider \& J. Teitelbaum, "p-adic Fourier theory", Doc. Math. 6 (2001), p. 447-481 (electronic).

[11] E. DE Shalit \& E. ICEland, "Integer valued polynomials and Lubin-Tate formal groups", J. Number Theory 129 (2009), no. 3, p. 632-639.

[12] J. T. TAтE, "p-divisible groups", in Proc. Conf. Local Fields (Driebergen, 1966), Springer, Berlin, 1967, p. 158-183.

Ehud DE SHALIT

Hebrew University

Jerusalem, Israel

E-mail: deshalit@math.huji.ac.il 